\title{
Phox2B correlates with MYCN and is a prognostic marker for neuroblastoma development
}

\author{
XIAO-XUE KE ${ }^{1 *}$, DUNKE ZHANG $^{1 *}$, HAILONG ZHAO $^{1}$, RENJIAN HU $^{2}$, ZHEN DONG $^{1}$, \\ RUI YANG ${ }^{1}$, SHUNQIN ZHU ${ }^{1}$, QINGYOU XIA ${ }^{1}$, HAN-FEI DING ${ }^{3}$ and HONGJUAN CUI ${ }^{1}$ \\ ${ }^{1}$ Cell Biology Laboratory, State Key Laboratory of Silkworm Genome Biology, Southwest University, Chongqing 400716; \\ ${ }^{2}$ Department of Pharmaceutical Engineering, School of Pharmacy and Bioengineering, Chongqing University of Technology, \\ Chongqing 400050, P.R. China; ${ }^{3}$ Department of Biochemistry and Molecular Biology, Cancer Center, \\ Medical College of Georgia, Georgia Regents University, Augusta, GA 30912, USA
}

Received July 25, 2014; Accepted March 19, 2015

DOI: $10.3892 / \mathrm{ol} .2015 .3088$

\begin{abstract}
Neuroblastoma is the one of the most common extracranial childhood malignancies, accounting for $\sim 15 \%$ of tumor-associated deaths in children. It is generally considered that neuroblastoma originates from neural crest cells in the paravertebral sympathetic ganglia and the adrenal medulla. However, the mechanism by which neuroblastoma arises during sympathetic neurogenesis and the cellular mechanism that drives neuroblastoma development remains unclear. The present study investigated the cell components during neuroblastoma development in the tyrosine hydroxylase- $v$-myc avian myelocytomatosis viral oncogene neuroblastoma derived homolog (TH-MYCN) mouse model, a transgenic mouse model of human neuroblastoma. The present study demonstrates that paired-like homeobox $2 \mathrm{~b}(\mathrm{Phox} 2 \mathrm{~B})^{+}$neuronal progenitors are the major cellular population in hyperplastic lesions and primary tumors. In addition, Phox $2 \mathrm{~B}^{+}$neuronal progenitors in hyperplastic lesions or primary tumors were observed to be in an actively proliferative and undifferentiated state. The current study also demonstrated that high expression levels of Phox2B promotes neuroblastoma cell proliferation and xenograft tumor growth. These findings indicate that the proliferation of undifferentiated $\mathrm{Phox}_{2} \mathrm{~B}^{+}$neuronal progenitors is a cellular mechanism that promotes neuroblastoma development and indicates that Phox $2 \mathrm{~B}$ is a critical regulator in neuroblastoma pathogenesis.
\end{abstract}

Correspondence to: Professor Hongjuan Cui, Cell Biology Laboratory, State Key Laboratory of Silkworm Genome Biology, Southwest University, 2 Tiansheng Road, BeiBei, Chongqing 400716, P.R. China

E-mail: hcui@swu.edu.cn; hongjuan.cui@gmail.com

*Contributed equally

Key words: neuroblastoma development, paired-like homeobox 2b, TH-MYCN mice

\section{Introduction}

Neuroblastoma accounts for $\leq 10 \%$ of childhood malignancies and $\sim 5 \%$ of all pediatric oncology-associated mortality (1). It is the most common extra-cranial solid tumor in patients $\leq 15$ years of age and the most frequently diagnosed type of cancer during infancy (2). Neuroblastoma is a disease that develops in the sympathetic nervous system, and the majority of cases arise in the paravertebral sympathetic ganglia and the adrenal medulla (2), which are derived from trunk neural crest cells $(3,4)$. During embryonic development, neural crest cells originate from the ectodermal part of the embryo that arises from the dorsal region of the neural tube, migrate along a ventral route and aggregate at the dorsal aorta to form the primary sympathetic chain (3-5). A group of cells in the primary sympathetic chain migrates in a dorsal direction to give rise to the paravertebral sympathetic ganglia, which constitutes the secondary (definitive) sympathetic chains, while another group of cells migrate into the adrenal gland to develop into the adrenal medulla $(4,6)$.

Histologically, neuroblastomas form a heterogeneous group of tumors, ranging from tumors containing undifferentiated or poorly differentiated neuroblasts to those with fully differentiated sympathetic neurons $(2,7,8)$. This observation indicates that neuroblastoma occurrence may be the result of deregulation of sympathetic neurogenesis. The molecular mechanism of sympathetic neurogenesis regulation has been relatively well-characterized (9). A number of bone morphogenetic proteins (BMPs) are released by the dorsal aorta during early neural crest development. This BMP signaling results in upregulated expression of various transcription factors that are essential for neuronal differentiation $(10,11)$. Initially, the sympathetic neural crest cells express the pro-neural molecules mammalian achaete-scute homolog 1 (Mash-1) and paired-like homeobox 2b (Phox2B), which is one of the paired homeodomain factors. The activation of Mash1 and Phox2B results in upregulated expression of other transcription factors, including Hand-2, Phox 2A, and the zinc-finger proteins GATA2 and GATA3, to promote further neuronal differentiation (12-18). These transcription factors collaborate in a complex regulatory network to induce tyrosine hydroxylase (TH) and dopamine 
$\beta$-hydroxylase (DBH) expression and result in pan-neuronal and subsequent catecholaminergic differentiation into sympathetic ganglia (19). A critical question is at what point neuroblastomas arise during sympathetic neurogenesis. It is generally considered that neuroblastomas develop from primitive sympathetic progenitor cells $(2,20)$, but knowledge of their identity remains limited. Identification of the cells that develop into neuroblastomas will increase the understanding of the cellular mechanism of tumorigenesis and provide a potential therapeutic target.

The genomic amplification of $v$-myc avian myelocytomatosis viral oncogene neuroblastoma derived homolog (MYCN) is the most common genetic aberration that is associated with poor outcome in neuroblastoma $(21,22)$. MYCN amplification occurs in $\sim 20 \%$ of primary neuroblastoma cases and is strongly correlated with advanced-stage disease and failed treatment (23). Crucially, transgenic mice carrying human MYCN under control of the $\mathrm{TH}$ promoter develop neuroblastomas with high resemblance to human tumors (24), suggesting that aberrant activation of MYCN can drive neuroblastoma development. It has been reported that the TH-MYCN mice develop tumors that closely resemble the human disease, including tumor site, morphology and further genomic alterations (25). In the present study, a detailed examination of hyperplastic lesions and primary tumors in TH-MYCN mice was performed and the correlation between the pro-neural gene Phox2B and neuroblastoma progression was investigated. The results of the present study using TH-MYCN mice may elucidate the cellular basis of neuroblastoma initiation, progression and pathogenesis.

\section{Materials and methods}

Mice. Forty male MYCN-transgenic mice [129X1/SvJ-Tg (TH-MYCN) 41Waw] and forty of their wildtype 129X1/SvJ littermates (age, 2 weeks; weight, 7-9 g) were purchased from the National Cancer Institute Mouse Repository (Frederick, MD, USA). All pathology experiments were performed in the MYCN-transgenic mice (TH-MYCN) and their wild-type littermates. The eighteen female NOD/SCID (age, 4 weeks; weight, 15-18 g) mice used in xenograft assay were obtained from the Jackson Laboratory (Ben Harbor, ME, USA). Animals had access to commercial pellet feed and water ad libitum, and were kept in a temperature-controlled room $\left(22^{\circ} \mathrm{C}\right)$ and maintained under specific pathogen-free conditions in the animal facility of Southwest University (Chongqing, China). All animal experiments were pre-approved by the Institutional Animal Care and Use Committee of Southwest University.

Cell culture. Five human neuroblastoma cell lines were obtained from the American Type Culture Collection (Manassas, VA, USA). SK-N-DZ, SK-N-AS and SH-EP1 were maintained in Dulbecco's modified Eagle's medium (DMEM) supplemented with $10 \%$ fetal bovine serum (FBS), plus $1 \%$ penicillin and streptomycin (P/S). BE(2)-C and IMR-32 were cultured in a 1:1 mixture of DMEM:F12 (F12/DMEM) supplemented with 10\% FBS and $0.1 \mathrm{mM}$ non-essential amino acids plus $1 \% \mathrm{P} / \mathrm{S}$. The lentiviral packaging cell line 293FT was cultured in DMEM containing $10 \% \mathrm{FBS}, 1 \% \mathrm{P} / \mathrm{S}, 0.5 \mathrm{mg} / \mathrm{ml} \mathrm{G} 418,4 \mathrm{mM}$ L-glutamine,
$0.1 \mathrm{mM}$ MEM non-essential amino acids and $1 \mathrm{mM}$ MEM sodium pyruvate. The 293FT cell line, growth media, FBS and supplemental reagents were obtained from Invitrogen Life Technologies (Carlsbad, CA, USA). All cells were cultured at $37^{\circ} \mathrm{C}$ in a $5 \% \mathrm{CO}_{2}$ humidified incubator.

Lentiviral constructs and infection. The lentiviral constructs pLK0.1-puro-GFP and pLK0.1-puro-MYCN were used in overexpression studies. For downregulation of MYCN and Phox2B, the lentiviral constructs pLK0.1-puro-GFPsi, pLK0.1-puro-MYCNsi and pLK0.1-puro-Phox2Bsi(\#1/\#2) were obtained from Sigma-Aldrich (St. Louis, MO, USA). The lentiviral constructs were transfected into 293FT packaging cells using Lipofectamine 2000 reagent (Invitrogen Life Technologies). Virus-containing supernatants were harvested and titered, and then were used to infect target cells with $4 \mu \mathrm{g} / \mathrm{ml}$ polybrene (Santa Cruz Biotechnology, Inc., Dallas, TX, USA). One day subsequent to the final round of infection, the cells were cultured in the presence of $2 \mu \mathrm{g} / \mathrm{ml}$ puromycin (Invitrogen Life Technologies) for 3 days and the drug-resistant cells were pooled.

Histology. For each genotype, 20 mice were sacrificed during the first 3 weeks after birth. The sympathetic ganglia were collected, fixed in $10 \%$ formalin (Sigma-Aldrich), then embedded in paraffin blocks (Sigma-Aldrich), together with the superior cervical ganglia (SCG) and the celiac ganglia. The paraffin-embedded samples were sectioned at $4 \mu \mathrm{m}$ and stained with hematoxylin and eosin (H\&E; Sigma-Aldrich; hematoxylin, $8 \mathrm{~min}$; eosin, $5 \mathrm{~min}$ ). Each ganglion was examined for the presence of hyperplastic lesions, which were defined as clusters of $\geq 30$ small blue round cells. TH-MYCN ( $n=20)$ samples and wild-type control $(n=20)$ were examined for the presence of tumors. For histological examination, the tumors were removed and stained with H\&E.

Immunohistochemistry. The sections were deparaffinized and rehydrated, then treated with $10 \mathrm{mmol} / \mathrm{l}$ citrate buffer $(\mathrm{pH} 6.0$; Sigma-Aldrich) at $95^{\circ} \mathrm{C}$ to retrieve antigens, and subsequently washed in phosphate-buffered saline (PBS). For immunohistochemical analysis, the endogenous peroxidase activity was quenched with $0.6 \% \mathrm{H}_{2} \mathrm{O}_{2}$ in methanol, and the sections were blocked with $10 \%$ normal goat serum (Beyotime Institute of Biotechnology, Haimen, China) and incubated sequentially with the following primary antibodies: Monoclonal mouse anti-human MYCN (1:100, clone AB-1; \#OP10; Oncogene Research Products, La Jolla, CA, USA); monoclonal mouse anti-human Ki67 (1:100, \#558615; BD Pharmingen, San Diego, CA, USA); monoclonal rat anti-human BrdU (1:200; \#ab6326; Abcam, Cambridge, UK) and monoclonal rabbit anti-human Phox2B (1:1,000; \#ab183741; Abcam); monoclonal mouse anti-rat TH (1:5,000; \#T1299; Sigma-Aldrich); polyclonal rabbit anti-rat TH $(1: 1,000$; \#AB152; Chemicon International, Temecula, CA, USA); and polyclonal rabbit anti-human Tuj1 (1:2,000; \#ab18207; Abcam). They were then incubated with secondary antibodies, biotinylated goat anti-rabbit (\#BA-1000), goat anti-rat (\#BA-9400) or anti-mouse (\#BA-9200) IgG (1:200; Vector Laboratories, Inc., Burlingame, CA, USA) and Vectastain ABC reagent (Vector Laboratories, Inc.). The immunostaining was visualized with 3,3'-diaminobenzidine (Sigma-Aldrich). The sections were then counterstained with 


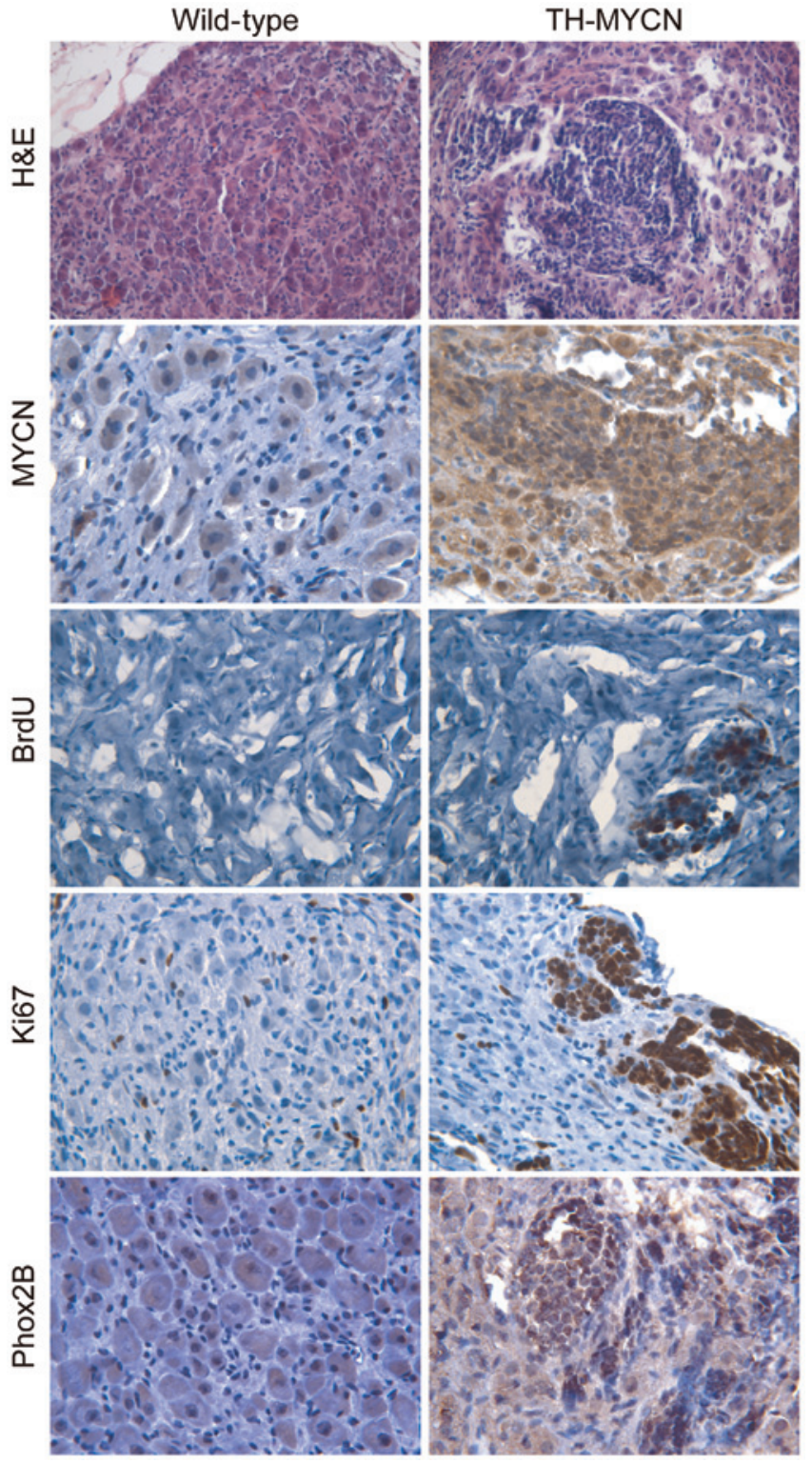

Figure 1. The TH-MYCN sympathetic ganglia were mainly composed of Phox $2 \mathrm{~B}^{+}$progenitor cells with proliferative activity. Sympathetic ganglia of TH-MYCN mice and their wild-type littermates were isolated and embedded in paraffin blocks, then stained with H\&E or MYCN, BrdU, Ki67 or Phox2B antibodies. H\&E and MYCN panels: Sympathetic ganglia of wild-type mice were mainly composed of large neurons and small glial cells and expressed undetectable levels of MYCN. In contrast, TH-MYCN sympathetic ganglia consisted of a large number of small blue round cells clustered together, and expressed high levels of MYCN. BrdU and Ki67 panels: The small population of proliferative cells in wild-type sympathetic ganglia expressed Ki67, but expressed undetectable levels of BrdU. The majority of hyperplastic cells in TH-MYCN sympathetic ganglia were proliferating cells marked by BrdU and Ki67. Phox2B panel: Hyperplasia of TH-MYCN sympathetic ganglia expressed markedly increased levels of Phox2B compared with their wild-type littermates. TH-MYCN, tyrosine hydroxylase- $v$-myc avian myelocytomatosis viral oncogene neuroblastoma derived homolog; Phox 2B, paired-like homeobox 2b; H\&E, hematoxylin and eosin.

hematoxylin before being examined using a light microscope (Eclipse 80i, Nikon, Tokyo, Japan).

Western blot analysis. Five human neuroblastoma cells were harvested and washed once with ice-cold PBS. Cell pellets were resuspended in SDS sample buffer (Thermo Fisher
Scientific, Life Technologies, Grand Island, NY, USA) and boiled for $10 \mathrm{~min}$, then centrifuged at $1500 \mathrm{x} \mathrm{g}$ for $10 \mathrm{~min}$. A total of $60 \mu \mathrm{g}$ of each protein sample was separated on a $12 \%$ SDS-polyacrylamide gel electrophoresis (stacking gel, $80 \mathrm{~V}$; acrylamide gel, $100 \mathrm{~V}$; polyacrylamide solution obtained from Sigma-Aldrich), and transferred to a polyvinylidene fluoride membrane (EMD Millipore, Billerica, MA, USA), which was then probed with antibodies and visualized by enhanced chemiluminescence with a BeyoECL Plus kit (Beyotime Institute of Biotechnology, Haimen, China). The following primary antibodies were used: Monoclonal mouse anti-human MYCN (1:100; clone AB-1; \#OP10; Oncogene Research Products), monoclonal mouse anti-human Mash1 (1:100; clone 24B72D11.1; \#556604; BD Pharmingen), monoclonal rabbit anti-human Phox2B (1:1,000; \#ab183741; Abcam), and monoclonal mouse anti-human $\alpha$-tubulin (1:2,000; B-5-1-2; \#T6074; Sigma-Aldrich). Horseradish peroxidase-conjugated goat anti-mouse (\#074-1806) and goat anti-rabbit (\#074-1506) IgG (1:20,000; KPL, Inc., Gaithersburg, MD, USA) were used as secondary antibodies.

Cell proliferation assays. Human neuroblastoma cells [BE(2)-C, SK-N-DZ and IMR-32] were seeded in 96-well culture plates. Cell proliferation was analyzed using a Cell Counting kit 8 (CCK-8; Beyotime Institute of Biotechnology). Briefly, $20 \mu \mathrm{l} \mathrm{CCK-8} \mathrm{was} \mathrm{added} \mathrm{into} 200 \mu \mathrm{l}$ medium in each well and the cells were incubated at $37^{\circ} \mathrm{C}$ for $2 \mathrm{~h}$. The absorbance was measured at a wavelength of $450 \mathrm{~nm}$ using a Model 550 microplate reader (Bio-Rad Laboratories, Inc., Hercules, CA, USA).

Soft agar clonogenic assay. A total of $1 \times 10^{3}$ cells [BE(2)-C-GFPsi, BE(2)-C-Phox 2Bsi\#1 and $\mathrm{BE}(2)-\mathrm{C}-\mathrm{Phox} 2 \mathrm{Bsi} \# 2]$ were mixed in $0.3 \%$ Noble agar (Sigma-Aldrich) in DMEM supplemented with 10\% FBS, and plated into 6 -well plates containing a solidified bottom layer $(0.6 \%$ Noble agar in the same growth medium). Colonies were stained with $5 \mathrm{mg} / \mathrm{ml}$ MTT (Sigma-Aldrich) after 21 days, and images were captured using a microscope (Olympus IX71; Olympus, Tokyo, Japan) and a scanner (Epson Perfection V850 Pro; Epson, Nagano, Japan). The results were recorded and analyzed statistically.

Xenograft assay. Female NOD/SCID mice of 4 weeks of age were maintained under specific pathogen-free conditions. For tumorigenic assays, $1 \times 10^{6}$ cells $[\mathrm{BE}(2)-\mathrm{C}, \mathrm{SK}-\mathrm{N}-\mathrm{DZ}$ and IMR-32] were suspended in $200 \mu \mathrm{l}$ serum-free DMEM and were injected subcutaneously into the flanks. The tumor growth was measured by caliper and the tumor volume was calculated with the formula $4 / 3 \pi r^{3}$, in which ' $r$ ' is the radius of the tumor. Three weeks following injection, the mice were sacrificed by cervical dislocation, and the xenograft tumors were immediately removed, weighed and paraffin-embedded.

Quantification and statistical analysis. All observations were confirmed by at least three independent experiments. Quantitative data are expressed as the mean \pm standard deviation. The two-tailed Student's t-test was performed using GraphPad Prism (version 6.0) for paired samples. $\mathrm{P}<0.05$ was considered to indicate a statistically significant difference. 


\section{Results}

Phox $2 B^{+}$neuronal progenitors are the major cellular component of hyperplastic lesions of TH-MYCN mice. To identify the cellular components during the early stage of neuroblastoma development, the SCG and celiac ganglia isolated from 3-week-old TH-MYCN mice were examined. Sympathetic ganglia were isolated from 3-week-old wild-type littermates as a control. The H\&E staining (Fig. 1) demonstrated that the sympathetic ganglia of wild-type littermates were composed of large sympathetic neurons and small glial cells. By contrast, multiple clusters of small blue round cells were present in TH-MYCN sympathetic ganglia. Immunohistochemical staining demonstrated that the hyperplastic lesions in sympathetic ganglia of TH-MYCN mice exhibited increased levels of MYCN compared with their wild-type littermates (Fig. 1).

Next, cell proliferation of hyperplastic lesions in sympathetic ganglia and ganglionic cells of control wild-type littermates were compared. Immunohistochemical staining was performed for two independent proliferation markers, BrdU (26) and Ki67 (27). As Fig. 1 demonstrates, the sympathetic ganglia of TH-MYCN mice contained a high level of $\mathrm{BrdU}^{+}$cells in hyperplastic clusters, while there was no detectable level of BrdU in the wild-type sympathetic ganglia. Although $\mathrm{Ki}^{+} 7^{+}$cells were evenly distributed in the wild-type sympathetic ganglia, no specific areas or zones of proliferation were observed. $\mathrm{Ki}_{67}{ }^{+}$cells were distributed in the non-hyperplastic region of TH-MYCN sympathetic ganglia. Notably, the majority of the hyperplastic cells in TH-MYCN sympathetic ganglia were $\mathrm{Ki} 67^{+}$cells.

Immunohistochemical staining was then performed to detect Phox2B, a marker of sympathetic neuronal progenitors (28). As demonstrated in Fig. 1, the majority of hyperplastic cells in the sympathetic ganglia of the TH-MYCN mice expressed Phox2B compared with samples from their wild-type littermates, which demonstrated low expression levels of Phox2B. Together, these results indicate that MYCN expression promotes the proliferation of $\mathrm{Phox} 2 \mathrm{~B}^{+}$neuronal progenitors to form hyperplastic lesions in sympathetic ganglia, which may lead to the initiation of neuroblastoma development.

Primary neuroblastoma tumors are composed predominantly of Phox $2 \mathrm{~B}^{+}$progenitor cells. To investigate whether MYCN-induced proliferation of Phox $2 \mathrm{~B}^{+}$neuronal progenitors promotes neuroblastoma progression, histological examination of primary neuroblastoma tumors from TH-MYCN mice was performed. The H\&E results demonstrated that the primary neuroblastoma tumors from the mice were almost entirely composed of small round blue cells (Fig. 2), which were histologically similar to the hyperplastic lesions in TH-MYCN sympathetic ganglia. The majority of the primary tumor cells stained positively for MYCN (Fig. 2) and the formation of nests or lobules surrounded by thin fibrovascular septa was observed, which are characteristics of human neuroblastomas with MYCN amplification (29). Next, the proliferation status of primary neuroblastoma tumors from TH-MYCN mice was investigated. The vast majority of primary tumor cells expressed Ki67, indicating that they were an actively proliferating population. Notably, the majority of the primary tumor cells expressed Phox2B, which was morphologically similar to the hyperplastic lesions observed in the sympathetic ganglia of the TH-MYCN mice, indicating that the primary tumor cells were in an undifferentiated state. These data demonstrated that proliferative $\mathrm{Phox} 2 \mathrm{~B}^{+}$neuronal progenitors were the major cellular component of primary neuroblastoma tumors from TH-MYCN mice. The results also indicated that the proliferation of undifferentiated $\mathrm{Phox} 2 \mathrm{~B}^{+}$neuronal progenitors may be a major cellular mechanism that promotes neuroblastoma progression.

Hyperplasia in TH-MYCN mice is mainly composed of undifferentiated progenitor cells. To confirm that the Phox $2 \mathrm{~B}^{+}$ neuronal progenitors were in an undifferentiated state, the tissue sections were immunostained for markers of mature sympathetic neurons. Fig. 3 demonstrates the morphology of mature sympathetic neurons from wild-type vs. TH-MYCN mice. In early postnatal sympathetic ganglia of wild-type mice, the majority of large sympathetic neurons expressed Tuj1, a marker for neuronal cells (30). A large number of sympathetic neurons in wild-type mice also expressed $\mathrm{TH}$, a late marker for sympathetic noradrenergic neurons (30). Similarly, cells in the non-hyperplastic region of TH-MYCN sympathetic ganglia expressed Tuj1 and TH. In contrast, in hyperplastic lesions of TH-MYCN sympathetic ganglia, almost all cells were small blue round cells, displayed an undifferentiated morphology and expressed no detectable levels of Tuj1 and TH. These results indicated an absence of neuronal differentiation in hyperplasia of TH-MYCN sympathetic ganglia. Thus, these data indicated that undifferentiated neuronal progenitors were the major cellular component of hyperplastic cells in TH-MYCN sympathetic ganglia. In addition, these findings indicate that expression of MYCN blocks neuronal progenitor differentiation into mature neurons and promotes the proliferation of Phox $2 \mathrm{~B}^{+}$ neuronal progenitors, leading to the formation of hyperplastic lesions and development of neuroblastoma.

High expression levels of Phox $2 B$ drive neuroblastoma cell proliferation and xenograft tumor growth. The data presented thus far in the present study indicate that MYCN-induced proliferation of undifferentiated $\mathrm{Phox} 2 \mathrm{~B}^{+}$neuronal progenitors may be an important cellular mechanism that promotes the formation of hyperplasia in TH-MYCN mice, resulting in neuroblastoma progression. In order to determine whether these findings in TH-MYCN mice are also present in the pathogenesis of human neuroblastoma, the role of Phox $2 \mathrm{~B}$ in the regulation of human neuroblastoma cell proliferation and xenograft tumor growth was investigated. The association between MYCN amplification and Phox2B expression in human neuroblastoma cells was determined. Immunoblotting demonstrated that the human neuroblastoma cell lines BE(2)-C, SK-N-DZ and IMR-32 are MYCN-amplified and express high levels of MYCN, however, they also express high levels of Phox2B (Fig. 4A). This result suggested that the expression level of Phox2B correlated with the MYCN expression level. In contrast, the human neuroblastoma SH-EP1 cell line expressed little or undetectable expression levels of MYCN and no detectable expression levels of Phox2B (Fig. 4A). Notably, cell proliferation analysis revealed 


\section{$H \& E$}
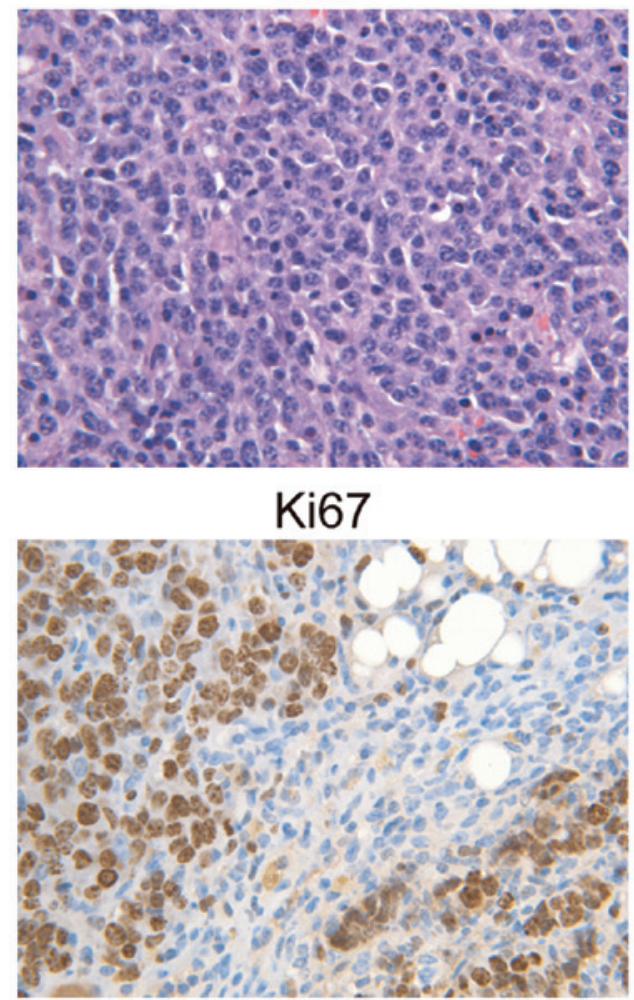

MYCN

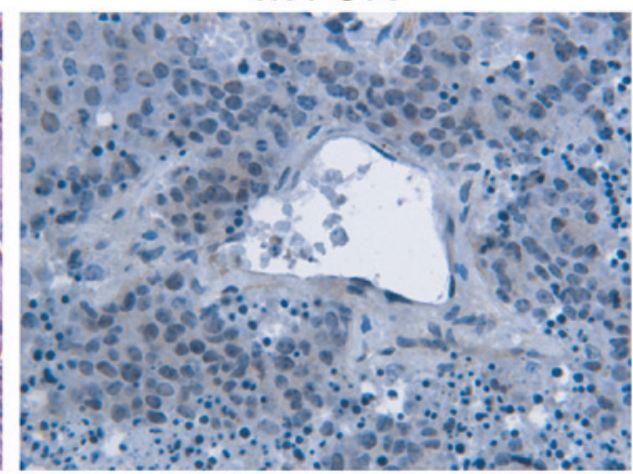

Phox2B

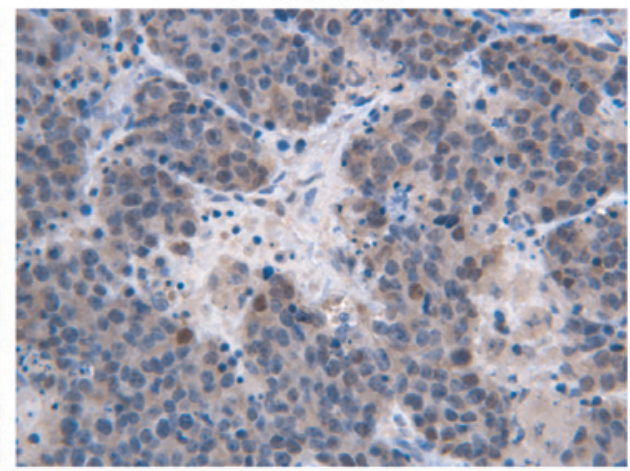

Figure 2. Primary tumors of TH-MYCN mice contained large numbers of proliferating Phox $2 \mathrm{~B}^{+}$progenitors. Sections of primary tumor from TH-MYCN mice were stained with H\&E or MYCN, Ki67, or Phox2B antibodies. Primary neuroblastoma tumors from TH-MYCN mice were almost entirely composed of small round blue cells and expressed high level of MYCN and Phox2B, with a large number of proliferating cells marked by Ki67. TH-MYCN, tyrosine hydroxylase- $v$-myc avian myelocytomatosis viral oncogene neuroblastoma derived homolog; Phox2B, paired-like homeobox 2b; H\&E, hematoxylin and eosin.


Figure 3. Hyperplasia of TH-MYCN mice predominantly consisted of undifferentiated progenitor cells. Sections of sympathetic ganglia from wild-type and TH-MYCN mice were immunostained for two neuron markers, Tuj1 and TH. The majority of cells in the wild-type sympathetic ganglia and the non-hyperplastic region of TH-MYCN sympathetic ganglia, displayed the morphology of mature neurons and expressed Tuj1 and TH. However, the hyperplastic lesion of TH-MYCN sympathetic ganglia stained negatively for Tuj1 or TH and displayed a small blue round cell morphology. TH-MYCN, tyrosine hydroxylase $v$-myc avian myelocytomatosis viral oncogene neuroblastoma derived homolog; Phox2B, paired-like homeobox 2b; H\&E, hematoxylin and eosin. 


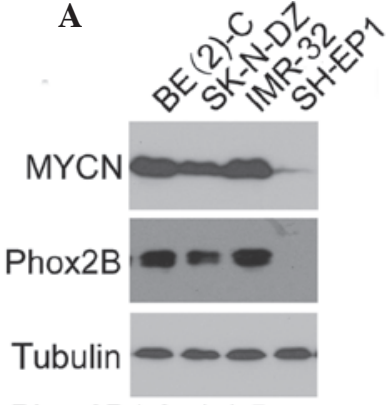

Phox2B1.3 11.5
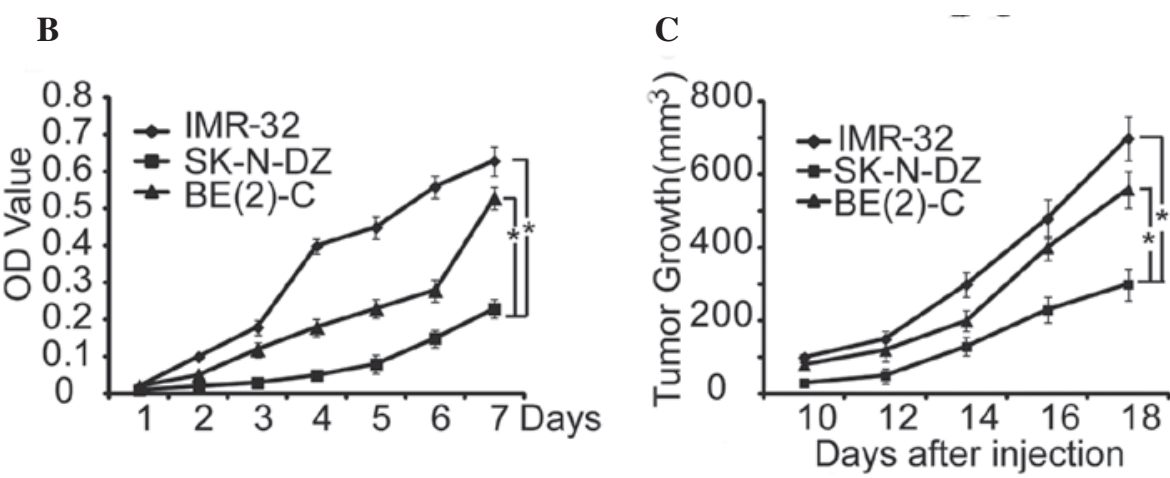

D

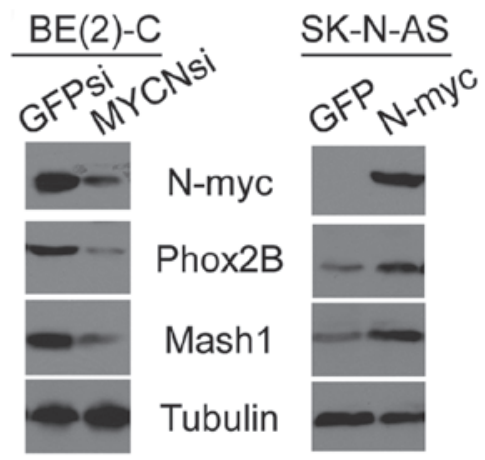

$\mathbf{F}$

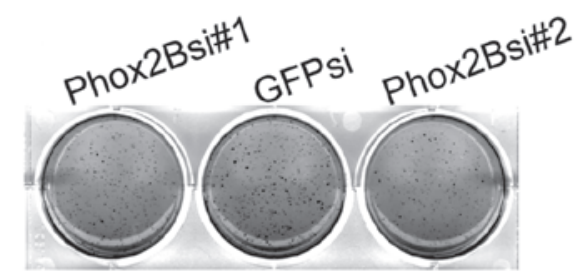

$\mathbf{E}$

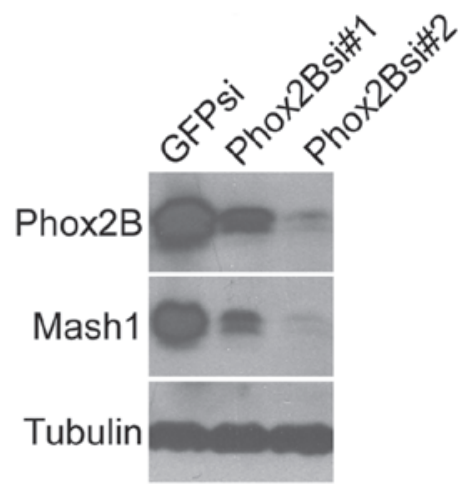

G

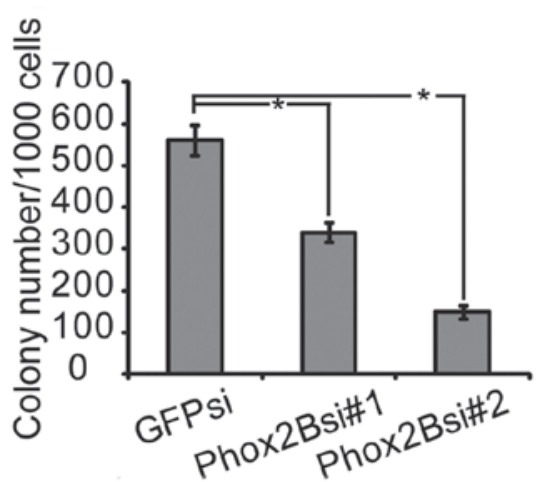

Figure 4. High level of Phox2B expression promoted human neuroblastoma cell proliferation and xenograft tumor growth. (A) Western blot analysis of MYCN and Phox 2B expression in 4 neuroblastoma cell lines. $\alpha$-Tubulin levels are presented as the loading control; the quantification of the expression level of Phox $2 B$ is normalized to that of the tubulin control. (B) Cell proliferation assay of 3 neuroblastoma cell lines, IMR-32, SK-N-DZ, and BE(2)-C, was performed using CCK-8. (C) Tumor growth in NOD/SCID mice injected with the 3 neuroblastoma cell lines, IMR-32, SK-N-DZ or BE(2)-C. (D) Western blot analysis of MYCN, Phox2B and Mash1 expression in BE(2)-C cells with MYCN knockdown by shRNA, or in SK-N-AS cells with MYCN overexpression. (E) Western blot analysis of Phox2B and Mash1 expression in BE(2)-C cells with Phox2B knockdown by shRNA. (F) Soft agar colony formation assay of BE(2)-C cells with Phox 2B knockdown by shRNA. (G) Colonies $\geq 0.5 \mathrm{~mm}$ or comprising $\geq 50$ cells were recorded. The data in $\mathrm{B}, \mathrm{C}$ and $\mathrm{G}$ are presented as the mean \pm standard deviation, obtained from 3 independent experiments. Statistical analysis was performed using two-tailed Student's t-test, ${ }^{*} \mathrm{P} \leq 0.05$. Phox $2 \mathrm{~B}$, paired-like homeobox 2b; MYCN, $v$-myc avian myelocytomatosis viral oncogene neuroblastoma derived homolog; CCK-8, cell counting kit 8; Mash1, mammalian achaete-scute homolog 1; shRNA, short hairpin RNA.

that cells with higher expression levels of Phox2B [IMR-32 and $\mathrm{BE}(2)-\mathrm{C}]$ demonstrated significantly greater levels of active proliferation compared with the SK-N-DZ cells $(\mathrm{P} \leq 0.05$; Fig. 4B), which were indicated to have lower levels of Phox2B expression.

Human neuroblastoma cells were injected into NOD/SCID mice to form xenograft tumors. Xenograft tumors derived from the neuroblastoma cells that expressed higher levels of Phox2B [IMR-32 and BE(2)-C] grew significantly faster than those derived from cells that expressed lower levels of Phox2B (SK-N-DZ) (P $\leq 0.05$; Fig. 4C). These findings indicate that Phox 2B expression may drive neuroblastoma growth.

The association between high expression levels of Phox 2B and MYCN, and the stemness of neuroblastoma cells was investigated. The BE(2)-C cell line expressed the highest level of MYCN and was therefore selected to investigate the effects of the downregulation of MYCN expression. This was achieved by using a short hairpin RNA specific to MYCN. Immunoblotting demonstrated that the inhibition of MYCN expression in $\mathrm{BE}(2)-\mathrm{C}$ cells led to a significant downregulation of Phox2B and Mash1, another 
marker for neuronal progenitors or stem cells (31) (Fig. 4D). The human neuroblastoma cell line SK-N-AS carries a single copy of MYCN and expresses little or undetectable levels of MYCN, therefore overexpression of MYCN was performed in the SK-N-AS cells. This analysis demonstrated that the overexpression of MYCN in SK-N-AS cells promoted a marked upregulation of Phox2B and Mash1 (Fig. 4D). These findings indicate that high expression levels of Phox2B, accompanied with MYCN amplification, are important to the stemness of neuroblastoma cells. To confirm the critical function of Phox $2 \mathrm{~B}$ in neuroblastoma stemness, the expression of Phox $2 \mathrm{~B}$ was knocked down in $\mathrm{BE}(2)-\mathrm{C}$ cells. This demonstrated that knockdown of Phox2B markedly induced downregulation of Mash1 expression (Fig. 4E), demonstrating that Phox2B was efficient in the regulation of neuroblastoma stemness. A clonogenic assay in soft agar was performed in order to examine the self-renewal capacity of the neuroblastoma cells. The knockdown of Phox2B in neuroblastoma BE(2)-C cells significantly inhibited the formation of colonies in soft agar $(\mathrm{P} \leq 0.05$; Fig. 4F and G), indicating that the downregulation of Phox $2 \mathrm{~B}$ results in suppression of the neuroblastoma cell self-renewal. This observation indicates that Phox $2 \mathrm{~B}$ is a cellular regulator for the maintenance of neuroblastoma stemness and promotion of neuroblastoma tumor growth.

In conclusion, the present study demonstrated that Phox $2 \mathrm{~B}$ is a major cellular regulator for maintaining neuroblastoma stemness that promoted neuroblastoma tumor growth, and indicated that the proliferation of undifferentiated Phox $2 \mathrm{~B}^{+}$ neuronal progenitors is a cellular mechanism that promoted neuroblastoma progression in TH-MYCN mice.

\section{Discussion}

Neuroblastoma is a type of cancer that arises in early neural progenitors of the sympathetic nervous system. Approximately a third of neuroblastomas originate in the adrenal glands; neuroblastomas also originate in the sympathetic ganglia in the abdomen, adjacent to the spine in the neck or chest, or in the pelvis (32). However, the causes of the majority of neuroblastomas remain unknown. It appears that, during embryonic development, neuroblastoma develops when normal neural crest cells fail to become adrenal medulla cells or mature nerve cells (2). In normal sympathetic neurogenesis, BMP signaling released by the dorsal aorta induces the neural crest cells to express high levels of Mash1 and Phox2B; this activates the expression of Hand-2, Phox2A, GATA2 and GATA3, which consequently promotes further neuronal differentiation $(17,22)$. We hypothesized that disorderly regulation of these transcription factors expressed in neural crest cells may be a potential incentive for neuroblastoma formation. The present study demonstrates that Phox $2 \mathrm{~B}^{+}$neuronal progenitors are the major cellular component of hyperplasia and primary tumors of TH-MYCN mice, therefore providing evidence in support of the hypothesis above.

The oncogene MYCN is critical in the pathogenesis of neuroblastoma (33) and aids in the regulation of cell growth (34). Similarly to other oncogenes, alterations in MYCN expression levels can result in accelerated cell growth and division (35). Previous studies have reported that the genomic amplification of MYCN is the most consistent genetic aberration associated with poor outcome in neuroblastoma $(21,36)$ and the MYCN transgenic mouse develops neuroblastoma with high resemblance to human tumors (24). Therefore, the TH-MYCN transgenic mouse was used in the present study as an animal model of neuroblastoma development to investigate whether Phox 2B is a critical factor in neuroblastoma development. The present study demonstrates that neuroblastoma development in TH-MYCN mice starts with hyperplastic lesion formation in the sympathetic ganglia during early sympathetic neurogenesis. The hyperplastic lesions are mainly composed of actively proliferative $\mathrm{Phox} 2 \mathrm{~B}^{+}$neuronal progenitors, as demonstrated by their high immunoreactivity for the proliferation markers BrdU and Ki67. In addition, in TH-MYCN hyperplastic lesions, the majority of cells exhibited an undifferentiated morphology and did not express detectable levels of Tuj1 and TH, two markers of mature neurons, indicating that the majority of cells in TH-MYCN hyperplastic lesions are maintained in a progenitor state. Thus, the proliferation of undifferentiated Phox $2 \mathrm{~B}^{+}$neuronal progenitors may lead to hyperplasia formation in TH-MYCN mice. The present study also demonstrated that $\mathrm{Phox} 2 \mathrm{~B}^{+}$and $\mathrm{Ki}^{+} 7^{+}$ proliferative neuronal progenitors are the major component of primary tumors from TH-MYCN mice. This data indicates that $\mathrm{Phox} 2 \mathrm{~B}^{+}$neuronal progenitors may contribute to neuroblastoma development through the maintenance of its proliferative activity.

The present study may also provide insight into the pathogenesis of human neuroblastoma. A previous study demonstrated that Phox2B is a predisposing gene to hereditary neuroblastic tumors (37). In accordance with this finding, the present study demonstrated that high expression levels of Phox2B promoted the growth of xenograft tumors derived from human neuroblastoma cells. High expression levels of Phox2B also promoted neuroblastoma cell proliferation and was essential to maintain neuroblastoma stemness. The evidence presented in the current study indicated that Phox $2 \mathrm{~B}$ may be critical for the pathogenesis of neuroblastoma.

\section{Acknowledgements}

The present study was supported by the National Basic Research Program of China (grant no. 2012cb114603), the National Natural Science Foundation of China (grant no. 81201551), the Research Fund for the Doctoral Program of Higher Education of China (grant no. 20130182110003) and the Fundamental Research Funds for the Central Universities (grant nos. SWU111014 and XDJK2011C017).

\section{References}

1. Hsieh MH, Meng MV, Walsh TJ, Matthay KK and Baskin LS: Increasing incidence of neuroblastoma and potentially higher associated mortality of children from nonmetropolitan areas: analysis of the surveillance, epidemiology, and end results database. J Pediatr Hematol Oncol 31: 942-946, 2009.

2. Brodeur GM: Neuroblastoma: biological insights into a clinical enigma. Nat Rev Cancer 3: 203-216, 2003.

3. Baker CV and Bronner-Fraser M: The origins of the neural crest Part I: embryonic induction. Mech Dev 69: 3-11, 1997.

4. Mayor R and Theveneau E: The neural crest. Development 140: 2247-2251, 2013.

5. Anderson DJ: Molecular control of cell fate in the neural crest: the sympathoadrenal lineage. Annu Rev Neurosci 16: 129-158, 1993.

6. Unsicker K: The chromaffin cell: paradigm in cell, developmental and growth factor biology. J Anat 183: 207-221, 1993. 
7. Sano H, Bonadio J, Gerbing RB, et al: International neuroblastoma pathology classification adds independent prognostic information beyond the prognostic contribution of age. Eur J Cancer 42: 1113-1119, 2006.

8. Shimada H, Ambros IM, Dehner LP, et al: The International Neuroblastoma Pathology Classification (the Shimada system). Cancer 86: 364-372, 1999.

9. Tsarovina K, Schellenberger J, Schneider C and Rohrer H: Progenitor cell maintenance and neurogenesis in sympathetic ganglia involves Notch signaling. Mol Cell Neurosci 37: 20-31, 2008.

10. Shah NM, Groves AK and Anderson DJ: Alternative neural crest cell fates are instructively promoted by TGFbeta superfamily members. Cell 85: 331-343, 1996.

11. Reissmann E, Ernsberger U, Francis-West PH, Rueger D, Brickell PM and Rohrer H: Involvement of bone morphogenetic protein- 4 and bone morphogenetic protein-7 in the differentiation of the adrenergic phenotype in developing sympathetic neurons. Development 122: 2079-2088, 1996.

12. Vincentz JW, VanDusen NJ, Fleming AB, et al: A Phox2- and Hand2-dependent Hand1 cis-regulatory element reveals a unique gene dosage requirement for Hand2 during sympathetic neurogenesis. J Neurosci 32: 2110-2120, 2012.

13. Hirsch MR, Tiveron MC, Guillemot F, Brunet JF and Goridis C: Control of noradrenergic differentiation and Phox2a expression by MASH1 in the central and peripheral nervous system. Development 125: 599-608, 1998.

14. Lo L, Tiveron MC and Anderson DJ: MASH1 activates expression of the paired homeodomain transcription factor Phox $2 \mathrm{a}$, and couples pan-neuronal and subtype-specific components of autonomic neuronal identity. Development 125: 609-620, 1998.

15. Stanke M, Junghans D, Geissen M, Goridis C, Ernsberger U and Rohrer $\mathrm{H}$ : The Phox 2 homeodomain proteins are sufficient to promote the development of sympathetic neurons. Development 126: 4087-4094, 1999.

16. Tsarovina K, Pattyn A, Stubbusch J, et al: Essential role of Gata transcription factors in sympathetic neuron development. Development 131: 4775-4786, 2004.

17. Pattyn A, Morin X, Cremer H, Goridis C and Brunet JF: The homeobox gene Phox $2 b$ is essential for the development of autonomic neural crest derivatives. Nature 399: 366-370, 1999.

18. Lim KC, Lakshmanan G, Crawford SE, Gu Y, Grosveld F and Engel JD: Gata3 loss leads to embryonic lethality due to noradrenaline deficiency of the sympathetic nervous system. Nat Genet 25: 209-212, 2000.

19. Schneider C, Wicht H, Enderich J, Wegner M and Rohrer H: Bone morphogenetic proteins are required in vivo for the generation of sympathetic neurons. Neuron 24: 861-870, 1999.

20. Dyer MA: Mouse models of childhood cancer of the nervous system. J Clin Pathol 57: 561-576, 2004.

21. Seeger RC, Brodeur GM, Sather H, et al: Association of multiple copies of the $\mathrm{N}$-myc oncogene with rapid progression of neuroblastomas. N Engl J Med 313: 1111-1116, 1985.
22. Maris JM and Matthay KK: Molecular biology of neuroblastoma. J Clin Oncol 17: 2264-2279, 1999.

23. Cohn SL, Pearson AD, London WB, et al: INRG Task Force: The International Neuroblastoma Risk Group (INRG) classification system: an INRG Task Force report. J Clin Oncol 27: 289-297, 2009.

24. Weiss WA, Aldape K, Mohapatra G, Feuerstein BG and Bishop JM: Targeted expression of MYCN causes neuroblastoma in transgenic mice. EMBO J 16: 2985-2995, 1997.

25. Hansford LM, Thomas WD, Keating JM, et al: Mechanisms of embryonal tumor initiation: distinct roles for $\mathrm{MycN}$ expression and MYCN amplification. Proc Natl Acad Sci USA 101: 12664-12669, 2004.

26. Muñoz-Velasco I, Ortíz R, Echeverría OM, Escobar ML and Vázquez-Nin GH: Characterization of the pre-meiotic $\mathrm{S}$ phase through incorporation of BrdU during spermatogenesis in the rat. J Histochem Cytochem 61: 680-689, 2013.

27. Pathmanathan N and Balleine RL: Ki67 and proliferation in breast cancer. J Clin Pathol 66: 512-516, 2013

28. Dubreuil V, Hirsch MR, Jouve C, Brunet JF and Goridis $\mathrm{C}$ : The role of Phox $2 \mathrm{~b}$ in synchronizing pan-neuronal and type-specific aspects of neurogenesis. Development 129: 5241-5253, 2002

29. Shimada H, Stram DO, Chatten J, et al: Identification of subsets of neuroblastomas by combined histopathologic and N-myc analysis. J Natl Cancer Inst 87: 1470-1476, 1995.

30. Egawa $C$ and Kameda $Y$ : Innervation of the chicken parathyroid glands: immunohistochemical study with the TuJ1, galanin, VIP, substance P, CGRP and tyrosine hydroxylase antibodies. Anat Embryol (Berl) 191: 445-450, 1995.

31. Sommer L, Shah N, Rao M and Anderson DJ: The cellular function of MASH1 in autonomic neurogenesis. Neuron 15: 1245-1258, 1995.

32. Maris JM, Hogarty MD, Bagatell R and Cohn SL: Neuroblastoma. Lancet 369: 2106-2120, 2007.

33. Huang M and Weiss WA: Neuroblastoma and MYCN. Cold Spring Harb Perspect Med 3: a014415, 2013.

34. Adhikary S and Eilers M: Transcriptional regulation and transformation by Myc proteins. Nat Rev Mol Cell Biol 6: 635-645, 2005.

35. Hipp NI, Christner L, Wirth T, et al: MYCN and survivin cooperatively contribute to malignant transformation of fibroblasts. Carcinogenesis 35: 479-488, 2014.

36. Brodeur GM and Seeger RC: Gene amplification in human neuroblastomas: basic mechanisms and clinical implications. Cancer Genet Cytogenet 19: 101-111, 1986.

37. Bourdeaut F, Trochet D, Janoueix-Lerosey I, et al: Germline mutations of the paired-like homeobox 2B (PHOX2B) gene in neuroblastoma. Cancer Lett 228: 51-58, 2005. 